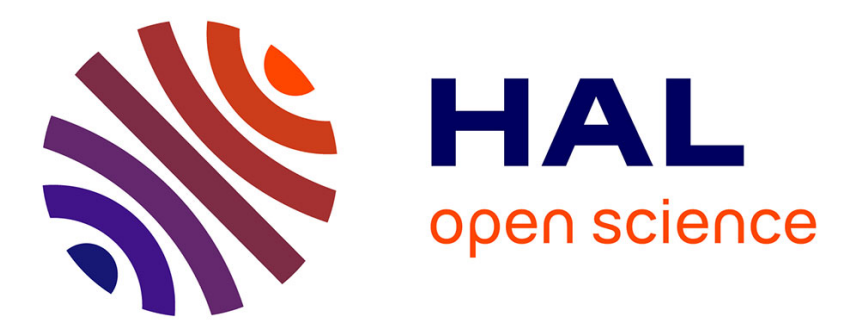

\title{
Complex Permittivity Determination From Far-Field Scattering Patterns
}

\author{
Christelle Eyraud, Jean-Michel Geffrin, Amelie Litman, Hervé Tortel
}

\section{To cite this version:}

Christelle Eyraud, Jean-Michel Geffrin, Amelie Litman, Hervé Tortel. Complex Permittivity Determination From Far-Field Scattering Patterns. IEEE Antennas and Wireless Propagation Letters, 2015, 14, pp.309 - 312. 10.1109/LAWP.2014.2362995 . hal-01279554

\section{HAL Id: hal-01279554 \\ https://hal-amu.archives-ouvertes.fr/hal-01279554}

Submitted on 31 Oct 2018

HAL is a multi-disciplinary open access archive for the deposit and dissemination of scientific research documents, whether they are published or not. The documents may come from teaching and research institutions in France or abroad, or from public or private research centers.
L'archive ouverte pluridisciplinaire HAL, est destinée au dépôt et à la diffusion de documents scientifiques de niveau recherche, publiés ou non, émanant des établissements d'enseignement et de recherche français ou étrangers, des laboratoires publics ou privés.

\section{(ㄷ)(1)}

Distributed under a Creative Commons Attribution| 4.0 International License 


\title{
Complex permittivity determination from far-field scattering patterns
}

\author{
C. Eyraud, J.-M. Geffrin, A. Litman and H. Tortel
}

\begin{abstract}
An accurate knowledge of the complex permittivity value of materials is compulsory when performing experimental electromagnetic applications. Unfortunately, these values are not so obvious to determine in practice. In the present work, we propose a novel approach for determining the complex dielectric constant of materials. This method combines free-space far-field scattering pattern measurements with a Bayesian procedure, which fully exploits the measurement uncertainties. Therefore, the measured values weighted according to their experimental accuracy are incorporated in the permittivity determination algorithm. In this paper, the samples are all shaped as spheres in order to benefit from efficient Mie scattered field computations. The dielectric properties of typical plastic samples are first determined and compared with values found in the literature, in order to assess the validity and the accuracy of the proposed methodology. A more "exotic" sample extracted from a microwave absorber, that is a polyurethane foam charged with carbon particles, is also analyzed.
\end{abstract}

Index Terms-Permittivity determination, Scattering field measurements, Bayesian procedure, Measurement uncertainties

\section{INTRODUCTION}

The knowledge of the complex permittivity values of materials is a prerequisite step for many kinds of electromagnetic applications. Indeed, an uncertainty in the dielectric constant knowledge of substrates can dramatically affect the functioning of microwave printed devices [1] such as the radiation patterns of printed antennas, RFID tags, etc. Similarly, in scattering phenomena, a local modification of the permittivity values will perturb the entire wave propagation process. In order to predict this propagation with the best accuracy, numerical calculations require a perfect knowledge of the electromagnetic properties as well as their repartitions in space [2] [3] [4]. Moreover, monitoring a change in the real and/or imaginary part of the complex permittivity might help to detect a variation of humidity, salinity, temperature, etc. Such monitoring systems require the development of robust inversion procedures, and at the same time, procedures to validate the accuracy of such imaging algorithms [5] [6].

In the microwave domain, several methods have already been proposed to derive permittivity measurements. Some of these techniques are based on appropriate resonators or cavities. Very accurate permittivity estimation can be obtained with such approaches [7] [8] [9], but these resonant methods are by nature narrow frequency-band. To overcome this drawback, methods using transmission line approaches have been developed using a coaxial cell [10] [11] [12] or a rectangular waveguide [13] [14]. These broadband methods

All the authors are with Aix Marseille Université, CNRS, Centrale Marseille, Institut Fresnel, UMR 7249, 13397, Marseille, France generally require a perfect contact between the sample and the conductors and thus a relative difficult sample preparation. To avoid this difficulty, permittivity characterization from partially filled arrangement [15] [16], [17] or from a cylindrical dielectric object placed in a rectangular waveguide [18] [19] [4] have been proposed. A counterpart of these methods is a more complex procedure to extract the permittivity value. Methods based on reflection/transmission measurements of a flat sample in free space have been also used [20] [21], in particular to perform high frequency permittivity estimation, but they need particular attention to overcome the multiple reflections and diffraction effects by the edge of the sample.

In this paper, we propose an original method, named scattering permittivity determination, for determining the dielectric characteristics of materials, which fully exploits the scattering phenomenon. Indeed, a sphere made from the material to be characterized is positioned inside an anechoic chamber. Its scattering pattern is then measured in far-field. This method is inspired from [22] [23] but without the constraints that the permittivity is constant on a frequency band [22] or that the spheres are small and assimilated to quadripoles [23]. An analytic calculation of the scattering pattern of a sphere is also performed thanks to Mie series. The permittivity is then determined using a Bayesian formulation allowing both to balance each measurement point according to its accuracy and to estimate the uncertainty on the retrieved permittivity. The procedure is further detailed in Section II. Section III is devoted to the presentation of the permittivity results. Several materials are analyzed: at first classical plastic samples and then a piece of a microwave absorber, a foam charged with carbon particles. Concluding remarks follow in Section IV.

\section{Procedure}

As the scattering pattern of an obstacle directly varies with the object electromagnetic characteristics, it can be used to derive interesting pieces of information on the scatterer. Among all available types of scatterers, we have only selected homogeneous spherical samples. We further assume the materials to be non-magnetic and isotropic. In that case, the scattered field only depends on five parameters: radius, position center, real and imaginary part of the permittivity and incident wave frequency. Moreover, with such a spherical target, the scattered field can be analytically computed with Mie series [24], preventing from supplementary numerical errors. Finally, interesting theoretical results can be directly exploited in that case. Indeed, the number of degree of freedom of the field scattered by a sphere is linked to the sphere size and 
to the illuminated wave frequency [25]. For a sphere of radius $R$ illuminated with a monochromatic-wave of wavelength $\lambda$ and observed on a circle enclosing the target, the number of degree of freedom of the scattered field in this plane called azimuthal plane - is equal to $2 q$ with $q=2 \pi R / \lambda$. As soon as $\frac{R}{\lambda} \geq \frac{1}{2 \pi}$, the number of information contained by the scattered field is thus superior to 2 . As only two unknowns the real $\varepsilon_{r}^{\prime}$ and imaginary $\varepsilon_{r}^{\prime \prime}$ part of the relative permittivity $\varepsilon_{r}$ - are searched for, the problem is rapidly overdetermined and a single incident illumination is sufficient.

\section{A. Field measurement}

The scattered fields have been measured with the spherical experimental setup available in the anechoic chamber of the CCRM-Marseille (Fig. 1). Both the experimental procedure and the post-processing treatments are described in [6] [26]. The electric fields scattered by the sphere are acquired on a circle enclosing the target - an angular sector is excluded due to mechanical constraints - keeping the scattered wavevectors and the incident wavevector in the same plane (Fig. 1). Due to the symmetry of the sphere, any plane which contains the sphere center can be chosen. A single polarization, $\perp \perp$ case - using the Bohren and Huffman definition - is selected [24].

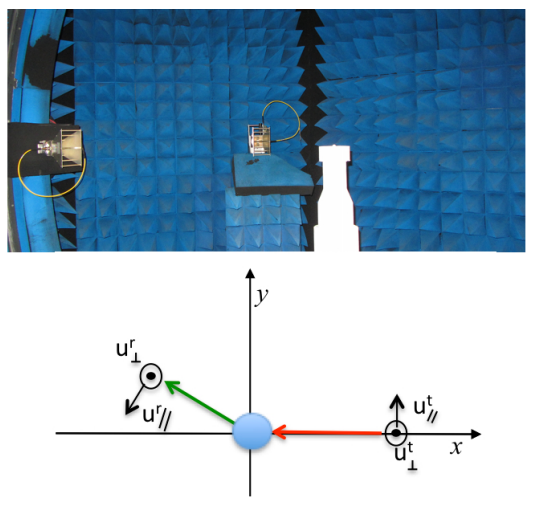

Fig. 1. Experimental setup: (top) photography of a small sphere in the anechoic chamber in the \|\| configuration case and (bottom) configuration sketch in the azimuthal $(x-y)$ plane.

\section{B. Misfit criterion}

The permittivity value of the sphere material is determined by comparing the measured scattered field $\mathbf{E}_{\mathbf{s}}^{\mathbf{m}}$ with the computed scattered fields $\mathbf{E}_{\mathbf{s}}^{\mathbf{c}}$ [27], at each frequency. The choice of the comparison criterion is of particular importance and is detailed hereafter while its exploitation for determining the permittivity is detailed in Section C.

As each measurement is compulsorily disturbed by some measurement errors, it is of great interest to take this knowledge into account in the comparison. As the systematic bias and the drift errors are considered to be totally eliminated from our measurements (see [6] [26]) and considering the positioning of the target to be accurate enough so as to neglect these errors, the measurements errors can be considered as pure random noise. In a previous study [6], this random noise has been fully characterized and the results have established that the deduced noise on the scattered field can be described by an additive complex random variable whose real and imaginary parts are normally distributed. The associated standard deviation $\sigma_{R e}$ (resp. $\sigma_{I m}$ ) on the real (resp. imaginary) part depends on the measured fields magnitude and on the frequency.

A Bayesian formulation allows one to include non-constant measurement errors in the inversion. Indeed, the determination of the relative permittivity value $\varepsilon_{r}$ from the scattered field measurement with the knowledge of the noise disturbing the measurement can be described by the following density of probability (see [28], [29] for example).

$$
p\left(\varepsilon_{r} \mid \mathbf{E}_{\mathbf{s}}^{\mathbf{m}}\right)=\frac{p\left(\varepsilon_{r}\right) p\left(\mathbf{E}_{\mathbf{s}}^{\mathbf{m}} \mid \varepsilon_{r}\right)}{p\left(\mathbf{E}_{\mathbf{s}}^{\mathbf{m}}\right)}
$$

Introducing no statistical information at all on the expected relative permittivity value, the probability $p\left(\varepsilon_{r}\right)$ follows an uniform law. Maximizing the distribution to obtain the permittivity value $\varepsilon_{r}$ with the knowledge of the measured scattered field $p\left(\varepsilon_{r} \mid \mathbf{E}_{\mathbf{s}}^{\mathbf{m}}\right)$ leads to the Maximum Likelihood solution. As there exist no noise correlation from one measurement point to another due to the measurement protocol, the involved criterion - calculated on the $N_{r}$ positions of the receivers - can be written as [30]

$$
\begin{aligned}
F^{B}\left(\varepsilon_{r}\right) & =\frac{1}{2 N_{r}} \sum_{r=1}^{N_{r}} f^{B}\left(\mathbf{r}_{r}\right) \\
f^{B}\left(\mathbf{r}_{r}\right) & =\frac{\operatorname{Re}\left[\mathbf{E}_{\mathbf{s}}^{\mathbf{m}}\left(\mathbf{r}_{r}\right)-\mathbf{E}_{\mathbf{s}}^{\mathbf{c}}\left(\mathbf{r}_{r}\right)\right]^{2}}{\sigma_{R e}^{2}\left(\mathbf{r}_{r}\right)}+\frac{\operatorname{Im}\left[\mathbf{E}_{\mathbf{s}}^{\mathbf{m}}\left(\mathbf{r}_{r}\right)-\mathbf{E}_{\mathbf{s}}^{\mathbf{c}}\left(\mathbf{r}_{r}\right)\right]_{(3)}^{2}}{\sigma_{I m}^{2}\left(\mathbf{r}_{r}\right)}
\end{aligned}
$$

If $\sigma_{R e}=\sigma_{I m}=1$, the criterion is nothing but the classical quadratic norm criterion $F^{Q}\left(\varepsilon_{r}\right)=\frac{1}{2 N_{r}} \sum_{r=1}^{N_{r}} f^{Q}\left(\mathbf{r}_{r}\right)$ with

$$
f^{Q}\left(\mathbf{r}_{r}\right)=\left|\mathbf{E}_{\mathbf{s}}^{\mathbf{m}}\left(\mathbf{r}_{r}\right)-\mathbf{E}_{\mathbf{s}}^{\mathbf{c}}\left(\mathbf{r}_{r}\right)\right|^{2}
$$

The shape of the curve $f^{B} / \sum f^{B}$ with the Bayesian approach

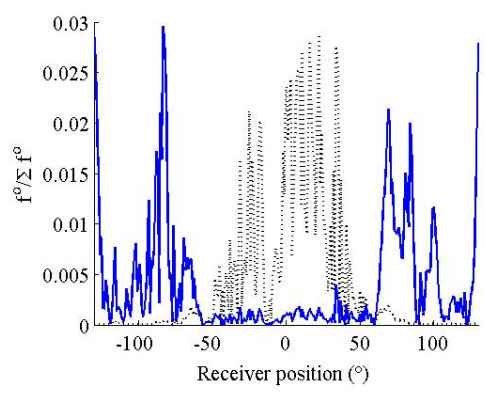

Fig. 2. Comparison of $f^{\bullet} /\left(\sum f^{\bullet}\right)$ with respect to the receiver positions (the $0^{\circ}$ position corresponds to forward scattering). (-) Bayesian criterion $(\bullet=B)$ and $(\ldots)$ Quadratic norm criterion $(\bullet=Q)$. These criteria have been calculated using the PMMA sphere at $10 \mathrm{GHz}$ (the associated permittivity results are presented in Fig. 4).

differs drastically from the usual quadratic norm criterion $f^{Q} / \sum f^{Q}$ (Fig. 2). Indeed, with the quadratic norm criterion, the measurement points corresponding to the forward zone are clearly predominant in the value of $F^{Q}$ and the contribution of the other angles are very weak, while with the Bayesian rule, each measurement point is correctly weighted with respect to its uncertainty. As for a given frequency, the weights depend 
on the magnitude of the total and the incident field which have important variations along the receiving angles, the weights greatly vary and have a key role in the value of $F$ and thus in the permittivity estimation.

\section{Permittivity values and uncertainties determination}

As Mie calculations do not require a heavy computational burden, it is possible to sample this criterion $F^{B}$ for various values of $\varepsilon_{r}^{\prime}$ and $\varepsilon_{r}^{\prime \prime}$, here with a sampling step equal to 0.01 (resp. 0.005) for the real (resp. imaginary) part. As it can be seen in Fig. 3, even if $\varepsilon_{r}^{\prime}$ and $\varepsilon_{r}^{\prime \prime}$ vary on a wide range, a single minimum is reached by the criterion $F^{B}$. All the other values are at least $15 \mathrm{~dB}$ higher than the minimum value. By detecting this value, we are able to obtain the complex permittivity without a priori information on the material, i.e, no information on the range of the permittivity value is needed. Each complex permittivity value found can also be associated with its uncertainty, defined as the variation which leads to a change of $3 \mathrm{~dB}$ in the criterion $F^{B}$ around the derived value.

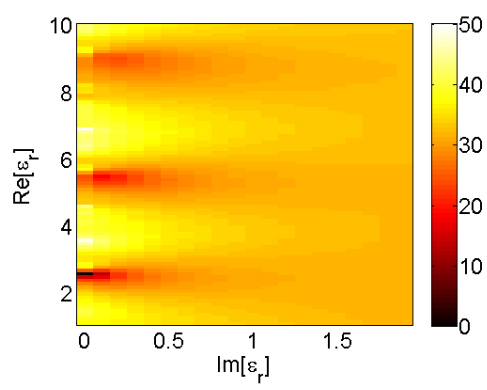

Fig. 3. Map in $\mathrm{dB}$ of $F^{B} /\left(\min F^{B}\right)$ for $\left(\varepsilon_{r}^{\prime}, \varepsilon_{r}^{\prime \prime}\right) \in[1,10] \times[0,2]$. This criterion has been calculated using the PMMA case at $10 \mathrm{GHz}$ (the associated permittivity results are presented in Fig. 4).

\section{RESULTS}

The procedure has been first validated with two materials which are well-known plastics: PolyMethylMethAcrylate (PMMA) and Poyacetal (PolyOxyMethylene). The sphere made in PMMA has a diameter of $50.75 \pm 0.05 \mathrm{~mm}$ and its permittivity is determined on the frequency band $[10-18] \mathrm{GHz}$ $(q \in[5.31-9.57])$. The results are presented in Fig. 4 and are within the uncertainties bars associated to the values found in the literature and obtained from resonant methods [8] [31]. The permittivity of the PolyOxyMethylene material has been determined from a sphere with a $50 \pm 0.3 \mathrm{~mm}$ diameter at $18 \mathrm{GHz}(q=9.43)$. This value is compared with permittivity values found in literature: (i) measured with a dielectric resonator but at a lower frequency $(11 \mathrm{GHz})[8]$ and (ii) measured with a coaxial cell at $18 \mathrm{GHz}$ [12] (Table I).

The interest of deriving a permittivity determination procedure is of course to be able to characterize as well uncommon material for which the permittivity value is not known. We first focus on the permittivity of the acrylate resin which is a material commonly employed nowadays in stereo-lithography. A spherical sample with a diameter of $25 \pm 0.1 \mathrm{~mm}$ is analyzed to establish its permittivity value at $20 \mathrm{GHz}(q=5.24)$ (see
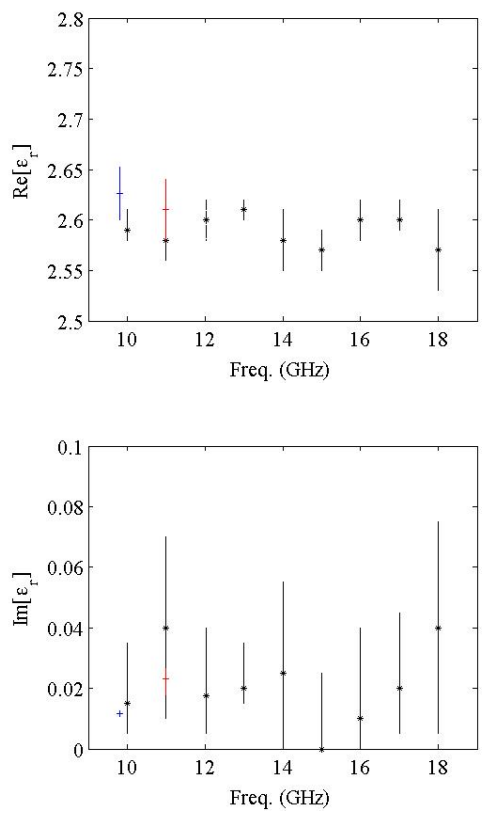

Fig. 4. Estimated complex permittivity values of the PMMA material with respect to frequency: $(*)$ sphere, $(+)[8]$ and $(+)$ [31].

TABLE I

ESTIMATED COMPLEX PERMITTIVITY VALUES OF THE POLYOXYMETHYLENE AND ACRYLATE RESIN SAMPLES.

\begin{tabular}{c|c|c|c|c|} 
Material & Method & Freq. & $\varepsilon_{r}^{\prime}$ & $\varepsilon_{r}^{\prime \prime}$ \\
\hline \multirow{3}{*}{ POM } & sphere & $18 \mathrm{GHz}$ & $2.85 \pm 0.05$ & $0 \pm 0.01$ \\
& {$[8]$} & $11 \mathrm{GHz}$ & $2.73 \pm 0.03$ & $0.014 \pm 0.004$ \\
& {$[12]$} & $18 \mathrm{GHz}$ & 2.8 & 0 \\
\hline \multirow{2}{*}{ Acrylate resin } & sphere & $20 \mathrm{GHz}$ & $2.85 \pm 0.15$ & $0.06 \pm 0.01$ \\
& {$[2]$} & $18 \mathrm{GHz}$ & 2.85 & 0.06
\end{tabular}

Table I). The deduced value has been successfully used for comparisons between simulated and measured fields when analyzing the scattering by two small spheres partially merging at $18 \mathrm{GHz}$ [2]. The second material is directly extracted from the absorbers which cover the walls in the anechoic chambers. The absorber is a foam composed of a polyurethane matrix charged with carbon particles [32]. From these absorbers, a sphere with a $69.3 \pm 0.1 \mathrm{~mm}$ diameter has been miled. Its permittivity has been determined on the frequency band $[1-8] \mathrm{GHz}(q \in[0.73,5.81])$ (see Fig. 5).

\section{CONCLUSION}

In this study, we have proposed a new broadband method for determining the complex permittivity value of solid materials. This procedure exploits the far-field scattering pattern of a sphere made with the under-test-material. This approach takes advantage of the analytic calculation of the scattered field which are easily available in the case of an isotropic and homogeneous sphere. Another advantage of the sphere is that its scattered field is completely ascertained when one knows its dimension, its position and its complex permittivity.

Our procedure takes into account the uncertainties of the measurements and makes best use of all the measurements thanks to a Bayesian approach, i.e., the measured values are weighted according to the experimental precision. In particular, this procedure allows to provide an a-posteriori error bar on each value which is linked to the uncertainty on 

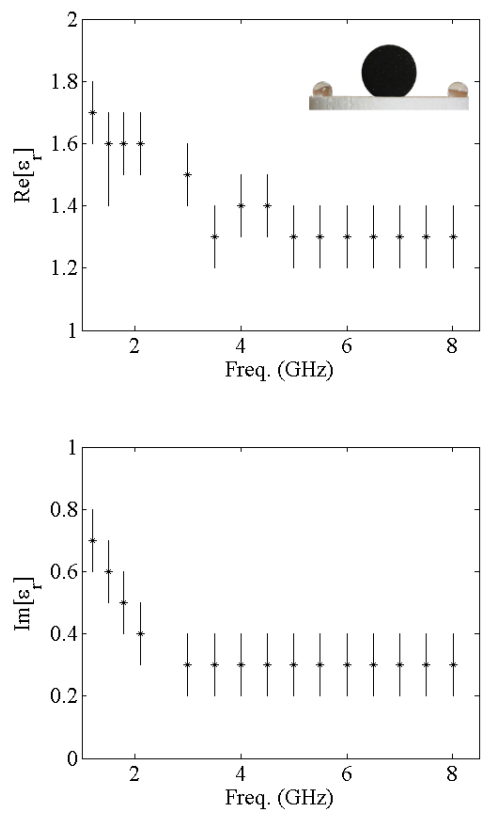

Fig. 5. Estimation of the complex permittivity values of a Siepel absorber with respect to frequency.

the scattered field measurements for this particular material and for a given frequency. The permittivity results have been found consistent with the results found in the literature for typical plastic materials. For these materials with very low losses, even if the uncertainty that we have determined on the imaginary part is larger than the one obtained with resonant methods, the uncertainty on the real part is of the same order - typically the relative error is around $1 \%$ to $2 \%$. The permittivity of more exotic materials, e.g., a foam charged with carbon particles (an absorber) has also been determined. In future work, this procedure will be further extended to determine the permittivity value of different kind of materials such as granular materials (sand, volcanic lava, etc) or liquids, which could be stored into a container and of different kind of forms.

\section{ACKNOWLEDGMENT}

The authors wish to acknowledge B. Lacroix from the CETHIL laboratory, Lyon for all the mechanics involving the fabrication of the absorber sphere and for providing the acrylate resin sphere.

\section{REFERENCES}

[1] P. Deffenbaugh, R. Rump and K. Church, Broadband Microwave Frequency Characterization of 3-D Printed Materials, IEEE Trans. Compon. Packag. Technol, 2156-3950, 2013

[2] P. Vaillon, J.-M. Geffrin, C. Eyraud, O. Merchiers, P. Sabouroux and B. Lacroix, A new implementation of a microwave analog to light scattering measurement device, J. Quant. Spectr. Radia. Trans., 112, 1753-60, 2011

[3] E. Heggy, E. Palmer, W. Kofman, S. Clifford, K. Righter and A. Hérique, Radar properties of comets: Parametric dielectric modeling of Comet 67P/ChuryumovGerasimenko, Icarus 211, 925, 2012

[4] M. Akhtar and M. Thumm, Measurement of complex permittivity of cylindrical objects in the E-plane of a rectangular waveguide, IEEE Trans. Geosci. Remote Sens., 51, 122-130, 2013

[5] J.-M. Geffrin, P. Sabouroux and C. Eyraud, Free space experimental scattering database continuation: Experimental setup and measurement precision, Inverse Problems 21, S117-130, 2005
[6] J.-M. Geffrin, C. Eyraud, A. Litman and P. Sabouroux, Optimization of a Bistatic Microwave Scattering Measurement Setup: From High to Low Scattering Targets, Radio Sci. 44, RS003837, 2009

[7] A. Parkash, J. Vaid and A. Mansingh, Measurement of dielectric parameters at microwave frequencies by cavity-perturbation technique, Trans. Microw. Theory Techn., 27, 791-795, 1979

[8] B. Riddle, J. Baker-Jarvis and J. Krupka, Complex Permittivity Measurements of Common Plastics Over Variable Temperatures, Trans. Microw. Theory Techn. 51, 727-733, 2003

[9] Z. Peng, J.-Y. Hwang and M. Andriese, Maximum Sample Volume for Permittivity Measurements by Cavity Perturbation Technique, IEEE Trans. Instrum. Meas., 63, 450-455, 2014

[10] A. Nicolson and G. Ross, Measurement of the intrinsic properties of materials by time-domain techniques, IEEE Trans. Instrum. Meas., 19, $791-795,1970$

[11] A. Gorriti and E. Slob, A New Tool for Accurate S-Parameters Measurements and Permittivity Reconstruction, IEEE Trans. Geosci. Remote Sens., 43, 1722-1735, 2005

[12] D. Ba and P. Sabouroux, EpsiMu, a toolkit for permittivity and permeability measurement in microwave domain at realtime of all materials: applications to solid and semisolid materials, Microw. Opt. Techn. Let. 52, 2643-2648, 2010

[13] W. Weir, Automatic measurement of complex dielectric constant and permeability at microwave frequency, Proc. IEEE 62, 33-36, 1974

[14] M. Akhtar, L. Feher and M. Thumm, A waveguide-based two-step approach for measuring complex permittivity tensor of uniaxial composite materials, IEEE Trans. Microw. Theory Techn., 54, 2011-2022, 2006

[15] R. York and R. Compton, An automated method for dielectric constant measurements of microwave structures, Microwave J. 38, 115-222, 1990

[16] J. Catala-Civera, A. Canos F. Penarand-Foix and E. Davos, Accurate determination of the complex permittivity of materials with transmission reflection measurements in partially filler rectangular waveguides, IEEE Trans. Microw. Theory Techn., 51, 16-24, 2003

[17] U. Hasar, Accurate complex permittivity inversion from measurements of a sample partially filling a waveguide aperture, IEEE Trans. Microw. Theory Techn., 58, 451-457, 2010

[18] K. Sarabandi, A technique for dielectric measurement of cylindrical objects in a rectangular waveguide, IEEE Trans. Instrum. Meas., 43, 793, 1984

[19] A. Nishikata, A swept-frequency measurement of complex permittivity and complex permeability of a columnar specimen inserted in a rectangular waveguide, IEEE Trans. Microw. Theory Techn., 455, 1554-1567, 2007

[20] M. Venkatesh and G. Raghavan, An overview of dielectric properties measuring techniques, Canadian Biosystems Eng. 47, 7.15, 2005

[21] M. Jilani, M. Rehman, A. Khan, M. Khan and S. Ali, A Brief Review of Measuring Techniques for Characterization of Dielectric Materials, ITEE J. 1, 2306, 2012

[22] R. Abbato, Dielectric Constant Measurements Using RCS Data, IEEE Proc., 112, 1095-1097, 1965

[23] J.S. Yu, L.J. Peters, Measurement of constitutive parameters using the Mie solution of a scattering sphere, IEEE Proc., 58, 876, 1970

[24] G. Borhen and D. Huffman, Absorption and Scattering of Light by Small Particles, Wiley, 1983

[25] O. Bucci and T. Isernia, Electromagnetic inverse scattering: retrievable information and measurement strategies, Radio Sci. 32, 2123-2137, 1997

[26] C. Eyraud, J.-M. Geffrin, A. Litman, P. Sabouroux and H. Giovannini, Drift correction for scattering measurements, Appl. Phys. Lett., 89, 244104, 2006

[27] H. Tortel, Localization and derivation of an optimal sphere for $3 D$ perfectly conducting objects, J. Electromagnet. Waves, 16, 771-791, 2002

[28] K. Mosegaard and A. Tarantola, Probabilistic Approach to Inverse Problems International Handbook of Earthquake and Engineering Seismology, Academic Press, 2002.

[29] A. Baussard, D. Premel and O. Venard, A bayesian approach for solving inverse scattering from microwave laboratory-controlled data, Inverse Problems, 17, 1659-69, 2001.

[30] C. Eyraud, A. Litman, A. Hérique and W. Kofman, Microwave imaging from experimental data within a Bayesian framework with realistic random noise, Inverse Problems, 25, 024005, 2009.

[31] J. Baker-Jarvis, M. Janezic, B. Riddle, C. Holloway, N. Paulter and J. Blendell, Dielectric and Conductor-Loss Characterization and Measurements on Electronic Packaging Materials, NIST Tech. Note 1520, 2001

[32] G. Dun, Modélisation et optimisation de chambres anéchoiques pour application CEM, PhD Thesis, ENSTB, 2008 